\title{
Originals
}

\section{Increased Platelet Aggregability in Diabetic Children: Relation to Serum Lipid and Fatty Acid Composition}

\author{
U. Ewald ${ }^{1}$, M. Kobbah ${ }^{1}$, B. Vessby ${ }^{2}$ and T. Tuvemo ${ }^{1}$ \\ Departments of ${ }^{1}$ Paediatrics and ${ }^{2}$ Geriatrics, University Hospital, Uppsala, Sweden
}

\begin{abstract}
Summary. Platelet aggregation was studied in 14 diabetic children with no signs of angiopathy and in 14 healthy matched control children. The magnitude of the platelet shape change after ADP stimulation was decreased in diabetic patients while the maximal aggregation after ADP and low dose collagen was significantly higher than in healthy control children. In 28 diabetic children the platelet shape change after ADP stimulation was positively correlated with the serum concentration of apolipoprotein A-I and negatively correlated with serum triglyceride concentration. The ratio between the fatty acids $20: 3 / 20: 4$ in cholesterol esters was strongly correlated with the relative incidence of irreversible aggregation $(p<0.001)$ and with the magnitude of the maximal aggrega-
\end{abstract}

tion $(p<0.01)$ after ADP stimulation $(3.3 \mu \mathrm{mol} / 1)$. The ratio between the polyunsaturated and saturated fatty acids in the triglyceride fraction was negatively correlated to the maximal aggregation after collagen stimulation $(10 \mathrm{mg} / \mathrm{l})$. This study shows that platelet aggregation is increased early in the course of diabetes in childhood. It suggests that the abnormalities in platelet aggregation pattern in diabetic patients are related to several of the lipid factors associated with an increased risk of atherosclerosis.

Key words: Lipids, fatty acids, juvenile diabetes mellitus, platelet aggregation, atherosclerosis.
In several studies during the last decade adult diabetic patients have been shown to have an increased platelet aggregability, particularly those with retinopathy and advanced vascular changes [1]. As more marked platelet changes were found in diabetic patients with more pronounced vascular disease, disturbed platelet function might be a consequence of the vascular disorder rather than the reverse [2-4]. Studies on diabetic children without clinically obvious vascular changes would seem to be of value in the analysis of this problem, especially as other factors influencing platelet function, e.g. smoking and drugs, can be avoided by choosing this study group.

The reason for the increased platelet aggregability in diabetes is unclear. Diabetic patients with hyperlipoproteinaemia have been shown to have more active platelets and incorporation of high concentrations of cholesterol into the platelet phospholipid membranes also increases platelet aggregability [5-7]. The fatty acid composition of platelet membrane phospholipids influences platelet function [8], and dietary enrichment with unsaturated fats changes platelet behaviour $[9,10]$. The serum fatty acid composition, especially the relative content of long-chain polyunsaturated acids, has been shown to be of importance for platelet function [11-14].
Recently, increased formation of thromboxanes - derivatives of long-chain polyunsaturated fatty acids - has been observed in platelets of diabetic patients [15].

Previously we have shown that the serum apolipoprotein (apo) A-I level is increased in diabetic children compared with normal subjects [16]. Apo A-I is regarded as a protective agent against atherosclerotic vascular changes [17]. The aim of the present study was to compare the platelet aggregability in diabetic children with that in healthy children and to correlate this aggregability to the lipid, apolipoprotein and fatty acid compositions of serum and serum fractions.

\section{Subjects}

Fourteen children aged 3-16 years with Type 1 (insulin-dependent) diabetes mellitus since $2-8$ years and their healthy peers of the same age and sex, who agreed to participate in the study as matched controls, comprised the study group for the comparison of platelet aggregation patterns. For the studies of the relationships between lipids and platelet aggregation 14 further diabetic children were added.

The diabetic children were all treated according to current Swedish schedules [18], which comprise two daily doses of monocomponent insulin and a regulated diet intended to supply $45 \%-50 \%$ of the energy intake from carbohydrates, $30 \%-33 \%$ from fat, $20 \%$ from pro- 
Table 1. Haemoglobin $A_{1}$, fasting plasma glucose and serum lipid concentrations in 28 diabetic and 14 healthy children

\begin{tabular}{lcc}
\hline & $\begin{array}{l}\text { Diabetic } \\
\text { children }\end{array}$ & $\begin{array}{l}\text { Control } \\
\text { children }\end{array}$ \\
\hline $\mathrm{HbA}_{\mathrm{I}}(\%)$ & $10.9 \pm 2.3$ & $6.5 \pm 0.4$ \\
Fasting plasma glucose $(\mathrm{mmol} / \mathrm{l})$ & $9.9 \pm 5.3$ & $4.1 \pm 0.3$ \\
Serum cholesterol $(\mathrm{mmol} / \mathrm{l})$ & $4.7 \pm 0.6$ & $4.6 \pm 1.6$ \\
Serum triglycerides $(\mathrm{mmol} / \mathrm{l})$ & $1.07 \pm 0.46$ & $0.7 \pm 0.27$ \\
\hline
\end{tabular}

Results expressed as mean $\pm \mathrm{SD}$

teins and to be rich in fibre and polyunsaturated fatty acids. Ophthalmoscopy was performed once a year by an ophthalmologist. No microaneurysms or other signs of retinopathy were found in any of the diabetic children. None had albuminuria. Indicators of diabetic control are given in Table 1 . None of the children had consumed any platelet inhibiting or other drugs during the preceding 2 weeks.

\section{Methods}

All blood specimens were taken before insulin injection and breakfast at $08.00-08.30 \mathrm{~h}$. An antecubital vein was punctured by a 19-G cannula and the first $\mathrm{ml}$ of blood was collected for lipid studies; $9 \mathrm{ml}$ of free flowing blood were then collected without venous stasis into a plastic syringe containing $1 \mathrm{ml}$ of $3.8 \%$ sodium citrate for platelet aggregation studies. The specimens from a diabetic child and the corresponding matched healthy control child were always taken in the same morning. The blood samples from one such pair were processed and analysed in parallel.

Lipids and apolipoproteins were determined as described previously [16]. The fatty acid compositions of cholesterol esters, triglyceride and phospholipids were analysed by gas-liquid chromatography [19]. The ratio of fatty acids $18: 2+20: 4+20: 5$ to fatty acids $14: 0$ $+16: 0+18: 0(\mathrm{P} / \mathrm{S}$ ratio) was calculated. For platelet aggregation studies the citrated blood was immediately prepared and platelet-rich plasma and platelet-poor plasma were obtained by centrifugation at $20^{\circ} \mathrm{C}$ at $150 \mathrm{~g}$ for $15 \mathrm{~min}$ and $1500 \mathrm{~g}$ for $10 \mathrm{~min}$, respectively. The platelets were counted in platelet-rich plasma on a Thrombocounter $\mathrm{C}$ (Coulter Electronics, Hialeh, Florida, USA) and the number adjusted to $300 \times 10^{9} / 1$ by the addition of platelet-poor plasma. The plateletrich plasma was kept in parafilm covered plastic tubes at room temperature and the aggregation studies were performed within $50-80 \mathrm{~min}$ after sampling. Platelet aggregation was studied by the turbidometric method of Born [20] on a Payton 600 Dual channel aggregometer (Payton Associates, Scarborough, Ontario, Canada) at $37^{\circ} \mathrm{C}$, with stirring at $900 \mathrm{rev} / \mathrm{min}$. The changes in optical densities were continuously monitored during and after addition of $50 \mu \mathrm{l}$ of aggregating agents in $250 \mu \mathrm{l}$ of specimen. Inducing agents used were adenosine diphosphate (ADP; Sigma, Stockholm, Sweden) 1.1, 2.2, 3.3 and $4.4 \mu \mathrm{mol} / \mathrm{l}$, and collagen Horm (Hormonchemie, München, FRG) 1.0 and $10.0 \mathrm{mg} / 1$ final concentrations in the cuvette. In the recordings after ADP stimulation, the presence of spontaneous aggregation and of reversible or irreversible aggregation was evaluated and the percentual changes in the optical densities (platelet-rich plas$\mathrm{ma}=100 \%$, platelet-poor plasma $=0 \%$ ) of the shape change, primary wave aggregation and maximal aggregation at 4 min were measured. After collagen stimulation the duration of the lag phase (the time until detectable decrease in optical density) and maximal aggregation obtained during 15 min were measured.

Statistical analysis was performed on an IBM 158-4341 computer at Uppsala University using the SAS program package (SAS Institute, Cary, N.C., USA). In comparisons between diabetic and control children, 14 matched pairs were tested by two-tailed paired Student's ttests. In the correlation studies all the twenty-eight diabetic children were included. The conventional 0.05 limit of significance was accepted in the correlations between lipids and platelet aggregation, where- as only correlations at probability levels of $<0.01$ were accepted in studies of the relations between fatty acids and the aggregation variables in order to avoid the effects of mass correlation.

\section{Results}

The platelet count in platelet-rich plasma did not differ significantly between diabetic and healthy control children.

No spontaneous aggregation was observed in any diabetic child or in any of the healthy control children. The occurrence of irreversible aggregation in the two groups were similar at all the ADP concentrations tested.

The magnitude of the platelet shape changes after ADP stimulation was lower in the diabetic patients at all concentrations tested and the differences were significant for ADP concentrations 3.3 ( $p<0.05$; Fig. 1) and $4.4 \mu \mathrm{mol} / 1(p<0.05)$.

The platelet aggregation induced after ADP stimulation was increased in the diabetic patients in comparison with that found in the normal children at all concentrations tested. The magnitude of the maximal aggregation at $4 \mathrm{~min}$ was significantly increased in the diabetic patients at ADP concentrations of $2.2(p<0.05)$ and $3.3 \mu \mathrm{mol} / 1(p<0.01 ;$ Fig. 1$)$. The change in optical density of the primary wave was significantly greater in the diabetic group after stimulation with ADP in concentrations of 1.1 and $3.3 \mu \mathrm{mol} / 1(p<0.01$ and $p<0.05$, respectively).

Low dose collagen $(1.0 \mathrm{mg} / 1)$ induced significantly increased $(p<0.01)$ aggregation in the diabetic children,

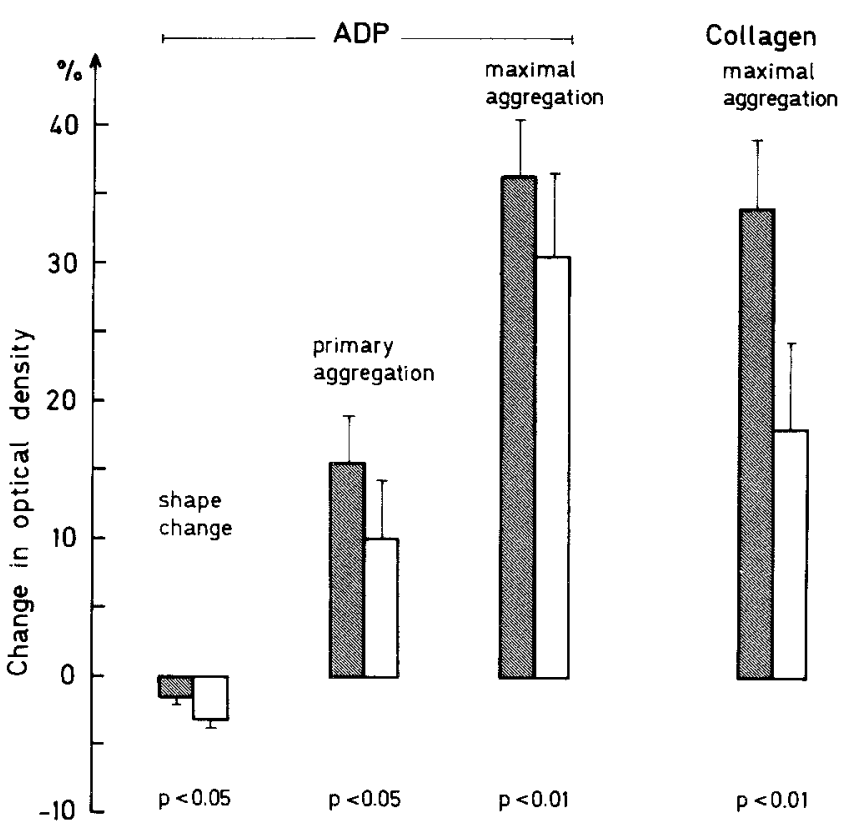

Fig. 1. Platelet shape change, primary wave aggregation and maximal aggregation after stimulation with $\operatorname{ADP}(3.3 \mu \mathrm{mol} / 1)$ and maximal aggregation after low dose collagen $(1.0 \mathrm{mg} / \mathrm{l})$ in 14 diabetic children $(\$)$ and in 14 normal healthy children $(\square)$. Means \pm SD are given 
Table 2. Correlation coefficients between platelet shape change after ADP stimulation and serum apo A-I concentration and between platelet shape change and serum triglyceride concentrations in 28 diabetic children

\begin{tabular}{lll}
\hline ADP stimulation & Apo A-I & Triglycerides \\
\hline $1.1 \mu \mathrm{mol} / 1$ & $r=0.43^{\mathrm{a}}$ & $r=-0.48^{\mathrm{a}}$ \\
$2.2 \mu \mathrm{mol} / 1$ & $r=0.40^{\mathrm{a}}$ & $r=-0.40^{\mathrm{a}}$ \\
$3.3 \mu \mathrm{mol} / 1$ & $r=0.40^{\mathrm{a}}$ & $r=-0.38$ \\
$4.4 \mu \mathrm{mol} / 1$ & $r=0.40^{\mathrm{a}}$ & $r=-0.35$ \\
\hline
\end{tabular}

${ }^{\mathrm{a}} p<0.05$

the mean maximal aggregation being doubled (Fig. 1). At a high dose of collagen $(10 \mathrm{mg} / \mathrm{l})$ there was no significant difference between diabetic and control children.

Within the group of 28 diabetic patients, there were significant positive correlations between the degree of platelet shape change at all ADP concentrations and the serum concentration of apo A-I $(p<0.05$; Table 2$)$. There was a negative correlation between fasting serum triglyceride levels and platelet shape change at concentrations of 1.1 and $2.2 \mu \mathrm{mol} / 1$ ( $p<0.05$; Table 2$)$.

The ratio between the fatty acids $20: 3$ to $20: 4$ in cholesterol esters was strongly associated with a high incidence of irreversible aggregation after stimulation with ADP in concentrations of 3.3 and $4.4 \mu \mathrm{mol} / 1$ $(r=0.61$ and 0.62 , respectively; $p<0.001$ ). This ratio was also correlated to the magnitude of the maximal aggregation after ADP at $3.3 \mu \mathrm{mol} / 1(r=0.52 ; p<0.01)$. The $\mathrm{P} / \mathrm{S}$ ratio in triglyceride was strongly negatively correlated to the maximal aggregation after stimulation with collagen $10 \mathrm{mg} / 1(r=-0.72 ; p<0.001)$. The concentration of the fatty acid $20: 5$ in the phospholipid fraction correlated negatively to the lag phase after collagen stimulation $(1 \mathrm{mg} / 1)(r=-0.54 ; p<0.01)$.

Platelet aggregation was not significantly correlated to the duration of the diabetic disorder in these children, nor was there any significant correlation to the degree of diabetic control as measured by haemoglobin $\mathrm{A}_{\mathrm{I}}$.

\section{Discussion}

The altered platelet function observed in the diabetic children of this study with an increased aggregability in response to ADP and collagen, is in agreement with findings in adult diabetic patients $[1,2,5]$. The experimental design used in this study for the comparison between platelet aggregability in diabetic children and matched healthy control children is expected to be sensitive but valid, as the blood samples are treated strictly in parallel during the processing of platelets and thereby excluding the influence of interassay variation. The absolute differences between the diabetic and the control children in this study, despite the statistical significance, are quantitatively small, with one exception, namely the difference in aggregability after low dose collagen stimulation, which was noteworthy.
These children have no clinical signs of microvascular disease and probably no atherosclerosis. This might indicate that the platelet aggregation disturbances are early diabetic changes, foregoing and possibly of pathogenetic importance for the development of the diabetic vascular disorder. However, we have also recently found that this same group of children with relatively well regulated diabetes of 2-8 years duration had significant changes of vascular reactivity [21]. Thus we are still unable to say whether the platelet changes precede the vascular disorders or vice versa, but we have demonstrated that functional changes in both systems exist at this stage of the disease.

The other major finding in this study was the decreased platelet shape change in diabetic children compared with healthy children. The reason for this reduction is not clear, but it might be a phenomenon in parallel with the decreased deformability of diabetic erythrocytes [22]. Membrane deformability might, in turn, depend on the fluidity of the phospholipid bilayer of the cell membrane, which is significantly influenced by fatty acid composition. The fatty acid composition in this group of children was, however, quite favourable, with high concentrations of polyunsaturated fatty acids [19].

Apolipoprotein A-I is believed to be protective against atherosclerosis in normal subjects [17]. It is therefore interesting to note that the diabetic children with the highest apo A-I levels had significantly higher values for platelet shape change, i.e. a more normal pattern. Apo A-I is believed to be a cholesterol acceptor decreasing the cholesterol content of cell membranes. This decrease results in a favourable phospholipid/cholesterol ratio, thus increasing the fluidity of phospholipid membranes. Apo A-I might therefore be a positive factor for platelet function in diabetes. This is of especial interest, as diabetic children have higher mean apo A-I values than healthy controls [16]. On the contrary, an increased serum triglyceride concentration, in some studies identified as a major cardiovascular risk factor [23], correlated negatively to platelet shape change at low ADP concentrations. These findings might indicate that a decreased change in platelet shape after ADP stimulation is of value as a predictor of the risk of atherosclerosis.

A high ratio between the fatty acids $20: 3 / 20: 4$ is regarded as a sign of essential fatty acid deficiency. A high ratio in cholesterol esters in these children was associated with an increased incidence of irreversible aggregation after moderate ADP stimulation $(3.3 \mu \mathrm{mol} / 1)$. A high intake of essential fatty acids in diabetic patients might reduce the tentative adverse effects of an increased tendency towards irreversible aggregation. In phospholipids, and certainly in phospholipid membranes, eicosapentaenoic acid, 20:5, is the substrate for thromboxane $\mathrm{A}_{3}$ and prostaglandin $\mathrm{I}_{3}$, a prostaglandin/ thromboxane system of special significance in Eskimos, in contrast to the situation in other ethnic groups, e.g. 
Danes [14]. It is interesting to note that this fatty acid, despite its very low absolute concentration, correlates significantly with the length of the lag phase after lowdose collagen stimulation. A high $\mathrm{P} / \mathrm{S}$ ratio in triglyceride, an indicator of high essential fatty acid intake during the last few days, was correlated to lower maximal aggregation after high dose collagen stimulation $(10 \mathrm{mg} / 1)$.

The abnormalities in platelet aggregation found in the diabetic children of this study thus seem to be less pronounced in patients with high serum concentrations of the cholesterol acceptor apo A-I and low concentrations of serum triglyceride. A favourable influence of some of the very long polyunsaturated essential fatty acids is also suggested. Changes of the lipid metabolism in this direction are in accordance with current recommendations for prevention of atherosclerosis in non-diabetic subjects, recommendations mainly based on data from epidemiological and intervention studies.

Acknowledgements. This study was supported by a grant from the Swedish Diabetes Association.

\section{References}

1. Silberbauer K, Schernthaner G, Sinzinger H, Freyler H (1981) Platelet aggregation and reversible platelet aggregates in Type Idiabetes staged by retinal fluorescein angiography. Atherosclerosis $40: 81-90$

2. Colwell JA, Halushka PV, Sarji K, Levine J, Sagel J, Nair RMG (1976) Altered platelet function in diabetes mellitus. Diabetes 25 (Suppl 2): 826-831

3. Burrows AW, Chavin SI, Hockaday TDR (1978) Plasma-thromboglobulin concentrations in diabetes mellitus. Lancet 1: 235-237

4. Preston FE, Ward JD, Marcola BH, Porter NR, Timperley WR (1978) Elevated B-thromboglobulin levels and circulating platelet aggregates in diabetic microangiopathy. Lancet 1:238-240

5. Micic J, Nesovic M, Djordjevic P, Micic D, Popovic, Jancic M (1979) Plasma triglyceride levels and platelet aggregation in diabetes mellitus. In: WaldhäusI W, Alberti KGMM (eds) Abstracts: 10 th Congress of the IDF. International Congress series 481. Excerpta Medica, p 409

6. Shattil SJ, Anaya-Galindo R, Bennet J, Colman RW, Cooper RA (1975) Platelet hypersensitivity induced by cholesterol incorporation. J Clin Invest 55: 636-643

7. Lees RS, Carvalho ACA (1978) Hypercholesterolemia and platelets. Adv Exp Med Biol 104: 301-308

8. Kalofoutis A, Lekakis J (1981) Changes of platelet phospholipids in diabetes mellitus. Diabetologia 21: $540-543$
9. Hornstra G (1973) Dietary fats and arterial thrombosis. Haemostasis $1973 / 74 ; 2: 21-52$

10. O'Brien JR, Etherington MD, Jamieson S, Vergroesen AJ, Ten Hoor F (1976) Effect of a diet of polyunsaturated fats on some platelet-function tests. Lancet ii: 995-997

11. Leading article (1980). Lipids, platelets, and atherosclerosis. Lancet $1: 464-465$

12. Needleman P, Whitaker MO, Wyche A, Watters K, Sprecher $H$, Raz A (1980) Manipulation of platelet aggregation by prostaglandins and their fatty acid precursors: Pharmacological basis for a therapeutic approach. Prostaglandins 19: 165-181

13. Seyberth HW, Oelz O, Kennedy T, Sweetman BJ, Danon A, Frölich JC, Heimberg M, Oates JA (1975) Increased arachidonate in lipids after administration to man: effects on prostaglandin biosynthesis. Clin Pharmacol Therapeut 18: 521-529

14. Dyerberg J, Bang HO (1979) Lipid metabolism, atherogenesis, and haemostasis in Eskimos: the role of the prostaglandin-3 family. Haemostasis 8: 227-233

15. Halushka PV, Rogers RC, Loadholt CB, Colwell JA (1981) Increased platelet thromboxane synthesis in diabetes mellitus. J Lab Clin Med 97: 87-96

16. Ewald U, Tuvemo T, Vessby B, Wålinder $O$ (1982) Serum apolipoproteins A-I, A-II and B in diabetic children and matched healthy controls. Acta Paediatr Scand 1:15-18

17. Avogaro P, Bittolo Bon G, Cazzolato G, Quinci GB (1979) Are apolipoproteins better discriminators than lipids for atherosclerosis? Lancet i: 901-903

18. The Swedish Study Group for Diabetes in Childhood (1981) Diabetes in childhood and adolescence, a suggested treatment program. triva-tryck, Linköping, Sweden

19. Ewald U, Gustafsson I-B, Tuvemo T, Vessby B (1982) Fatty acid composition of serum lipids in diabetic children and their matched healthy controls. Upsala J Med Sci 87: 111-117

20. Born GVR (1962) Aggregation of blood platelets by adenosine diphosphate and its reversal. Nature 194: 927-29

21. Ewald U, Tuvemo T, Rooth G (1981) Early reduction of vascular reactivity in diabetic children detected by transcutaneous oxygen electrode. Lancet i: $1287-1288$

22. Tillman W, Merten A, Lakomek M, Gahr M, Fiechtl J, Schröter W (1981) Deformability of red cells of diabetic children. Biblthca Anat 20: 166-169

23. Carlsson LA, Böttiger LE, Åhlfeldt P-E (1979) Risk factors for myocardial infarction in the Stockholm. Prospective Study. Acta Med Scand 206: 351-360

Received: 14 February 1983

and in revised form: 20 June 1983

Dr. T. Tuvemo

Department of Paediatrics

University Hospital

S-751 85 Uppsala

Sweden 